\title{
Clinical Features and Genetic Background of the Periodic Fever Syndrome with Aphthous Stomatitis, Pharyngitis, and Adenitis: A Single Center Longitudinal Study of 81 Patients
}

\author{
Daša Perko, ${ }^{1}$ Maruša Debeljak, ${ }^{2}$ Nataša Toplak, ${ }^{1,3}$ and Tadej Avčin ${ }^{1,3}$ \\ ${ }^{1}$ Department of Allergology, Rheumatology and Clinical Immunology, University Children's Hospital, University Medical Center, \\ Bohoričeva 20, 1000 Ljubljana, Slovenia \\ ${ }^{2}$ Unit for Special Laboratory Diagnostics, University Children’s Hospital, University Medical Center, Bohoričeva 20, \\ 1000 Ljubljana, Slovenia \\ ${ }^{3}$ Faculty of Medicine, University of Ljubljana, Vrazov trg 2, 1000 Ljubljana, Slovenia
}

Correspondence should be addressed to Tadej Avčin; tadej.avcin@kclj.si

Received 26 November 2014; Accepted 22 December 2014

Academic Editor: Nejat Akar

Copyright (C) 2015 Daša Perko et al. This is an open access article distributed under the Creative Commons Attribution License, which permits unrestricted use, distribution, and reproduction in any medium, provided the original work is properly cited.

PFAPA syndrome is the most common autoinflammatory disorder in childhood with unknown etiology. The aim of our study was clinical evaluation of PFAPA patients from a single tertiary care center and to determine whether variations of $A I M 2, M E F V$, NLRP3, and MVK genes are involved in PFAPA pathogenesis. Clinical and laboratory data of consecutive patients with PFAPA syndrome followed up at the University Children's Hospital, Ljubljana, were collected from 2008 to 2014. All four genes were PCR amplified and directly sequenced. Eighty-one patients fulfilled criteria for PFAPA syndrome, 50 (63\%) boys and 31 (37\%) girls, with mean age at disease onset of $2.1 \pm 1.5$ years. Adenitis, pharyngitis, and aphthae were present in $94 \%$, $98 \%$, and $56 \%$, respectively. Family history of recurrent fevers in childhood was positive in $78 \%$. Nineteen variants were found in $17 / 62(27 \%)$ patients, 4 different variants in NLRP3 gene in 13 patients, and 6 different variants in MEFV gene in 5 patients, and 2 patients had 2 different variants. No variants of clinical significance were found in $M V K$ and AIM2 genes. Our data suggest that PFAPA could be the result of multiple low-penetrant variants in different genes in combination with epigenetic and environmental factors leading to uniform clinical picture.

\section{Introduction}

Periodic fever syndromes (PFSs) are a wide group of autoinflammatory disorders, characterized by recurrent and seemingly unprovoked multisystemic inflammation in the absence of infection and autoantibodies formation $[1,2]$. Most hereditary PFSs are due to mutations in genes coding molecules of pyrin and tumor necrosis factor superfamilies both of which are directly involved in innate immunity [3].

Periodic fever with aphthous stomatitis, pharyngitis, and adenitis (PFAPA) represents the most common periodic fever syndrome of childhood with unknown actual incidence [4]. In contrast to monogenic periodic fever syndromes, the genetic defects in PFAPA have not yet been identified. This clinical entity is characterized by a sudden onset of fever which lasts for 3 to 6 days and spontaneously resolves afterwards. Fever is accompanied by aphthous stomatitis, pharyngitis, and cervical adenitis [5-7] with less common symptoms such as headache, rash, and gastrointestinal disturbances $[5,8]$. PFAPA episodes recur every 3 to 8 weeks. During the remission, patients are asymptomatic and show normal growth and development. Disease onset is usually before the age of five and generally resolves before puberty with no consequences for the patient [7-9]. No specific diagnostic test for PFAPA is currently available; therefore, the diagnosis is based only on diagnostic criteria [10].

Despite the fact that PFAPA is considered as a sporadic disease, many studies suggest that it might have a genetic origin. Syndrome has overlapping symptoms with other PFSs with known genetic cause [11] and, furthermore, recurrent 
fever in the family members of PFAPA patients has been reported in several studies $[10,12]$. In addition, genetic variants that are known to cause other autoinflammatory syndromes have been found in PFAPA patients $[9,13]$, but the impact of these genetic variants in PFAPA syndrome is still unknown.

Although the pathogenesis of PFAPA remains poorly understood, it is assumed that an environmental trigger in the context of immune abnormalities of the host may trigger disease episodes through activation of innate immune system, possibly via "absent in melanoma 2" (AIM2) inflammasome in monocytes, which leads to activation and secretion of IL$1 \beta$, induction of Th1-chemokines, and subsequent migration of T-lymphocytes into periphery [5].

AIM2 is a 343 amino acids long interferon inducible protein which plays a role as a receptor for cytosolic DNA. It consists of two subunits: C-terminal HIN-200 domain which binds double stranded DNA and N-terminal pyrin domain which interacts with pyrin domain of apoptosis-associated speck-like protein (ASC). ASC recruits procaspase-1 to the inflammasome complex which ultimately leads to activation of cytokines (IL-1 $\beta$ and IL-18) and inflammation [14-16]. It has been shown that AIM2 gene is overexpressed in PFAPA patients during flare compared to PFAPA patients in remission and healthy controls [5].

Given that IL-1 $\beta$ pathway is involved in the PFAPA pathogenesis, it is likely that AIM2 gene could also play an important role in this syndrome.

The aim of our study was to evaluate clinical features and molecular genetic background in a large, well-characterized cohort of patients with PFAPA syndrome from a single tertiary care center. In particular, we aimed to analyze involvement of AIM2 gene in the pathogenesis of PFAPA, which has not been previously tested in patients with PFSs. A second goal was to evaluate possible association of $M E F V$, NLRP3, MVK, and AIM2 gene variants with clinical features of the syndrome.

\section{Methods}

2.1. Participants. Clinical and laboratory data of 81 consecutive patients with PFAPA syndrome followed up at the University Children's Hospital, Ljubljana, were collected from January 2008 to June 2014. All participants included in the study fulfilled the clinical criteria for PFAPA syndrome [8]. Ethnicity and family history of recurrent fever, tonsillectomy, and repeated tonsillopharyngitis in family members were also obtained.

Samples from 100 apparently healthy adult subjects of Slovenian ethnicity that donated their DNA for research purposes were included in the study as healthy controls.

2.2. Ethics Committee Approval. The parents of each child included in the study were informed about the aim of the study and asked for written informed consent for inclusion in the study. The study was approved by the Ethics' Committee of the Republic of Slovenia and was conducted according to the principles of the Helsinki Declaration.
2.3. Samples and Blood Work. PFAPA patients had blood work performed during routine venipuncture initially and/or at the followup visits including complete blood count (CBC), C-reactive protein (CRP), erythrocyte sedimentation rate (ESR), serum immunoglobulin levels (IgA, IgG, IgM, and IgD), antinuclear antigen antibodies (ANA), serum amyloid A (SAA), antistreptolysin O titer (ASO), classical and alternative complement pathway activity (CH50, APH50), and lymphocyte subsets.

2.4. DNA Isolation and Primer Selection. DNA was isolated from 62 patients with PFAPA syndrome. Peripheral blood $(5 \mathrm{~mL})$ for DNA isolation was taken together with blood for routine laboratory work. DNA isolation was performed using the FlexiGene isolation kit (Qiagen, Germany), according to the recommended protocol. DNA was stored at $4^{\circ} \mathrm{C}$ prior to further molecular analyses.

PCR primers (Eurofins MWG Operon, Germany) were designed according to the established laboratory protocol (sequences are available upon request), covering whole coding regions and intron/exon boundaries of all four genes (MEFV, NLRP3, MVK, and AIM2) including promoter, $5^{\prime} \mathrm{UTR}$, and $3^{\prime} \mathrm{UTR}$ regions of AIM2 gene.

2.5. Polymerase Chain Reaction. PCR was performed on GeneAmp PCR System 9700 (Applied Biosystems, USA) using the AmpliTaq polymerase (Applied Biosystems, USA) for the whole NLRP3 gene, part of MEFV gene (exons 1, 3, 4, and 6-10) and part of $M V K$ gene (exons 2-7, 9, and 11), AmpliTaq Gold polymerase (Applied Biosystems, USA) for exons 2 and 5 of $M E F V$ gene, and GoTaq G2 Flexi DNA polymerase (Promega, USA) for the whole AIM2 gene and exons 8 and 10 of $M V K$ gene.

The reactions with both AmpliTaq polymerases were set up using $100 \mathrm{ng}$ of double stranded DNA, $0.2 \mathrm{mM}$ of each of the dNTPs, $0.4 \mu \mathrm{M}$ of primers, $1.5 \mathrm{mM}$ or $1.8 \mathrm{mM}$ (for AmpliTaq Gold) of $\mathrm{MgCl}_{2}, 1 \mathrm{x}$ reaction buffer, and $0.75 \mathrm{U}$ of polymerase in a final reaction volume of $25 \mu \mathrm{L}$. $3.4 \mu \mathrm{L}$ of $40 \%$ dimethyl sulfoxide was added when AmpliTaq Gold was used. The thermocycling procedure was conducted according to the following protocol: denaturing step at $95^{\circ} \mathrm{C}$ for $5 \mathrm{~min}$ (10 min for AmpliTaq Gold) followed by 35 cycles of $94^{\circ} \mathrm{C}$ for $30 \mathrm{~s}$, annealing step at $58^{\circ} \mathrm{C}-60^{\circ} \mathrm{C}$ (adjusted for each set of primers individually) for $30 \mathrm{~s}$, extension step at $72^{\circ} \mathrm{C}$ for $40 \mathrm{~s}$, and final extension at $72^{\circ} \mathrm{C}$ for $7 \mathrm{~min}$.

The reaction with GoTaq G2 Flexi DNA polymerase was set up using $100 \mathrm{ng}$ of double stranded DNA, $0.2 \mathrm{mM}$ of each of the dNTPs, $0.32 \mu \mathrm{M}$ of primers, $2 \mathrm{mM}$ of $\mathrm{MgCl}_{2}$, $1 \mathrm{x}$ green buffer, and $0.75 \mathrm{U}$ of polymerase in a final reaction volume of $25 \mu \mathrm{L}$. The thermocycling procedure consisted of initial denaturing step at $95^{\circ} \mathrm{C}$ for $2 \mathrm{~min}$ followed by 35 cycles of $94^{\circ} \mathrm{C}$ for $30 \mathrm{~s}$, annealing step at $60^{\circ} \mathrm{C}$ or $61^{\circ} \mathrm{C}$ (adjusted for each set of primers individually) for $30 \mathrm{~s}$, extension step at $72^{\circ} \mathrm{C}$ for $40 \mathrm{~s}$, and final extension at $72^{\circ} \mathrm{C}$ for $7 \mathrm{~min}$. The PCR products were subjected to electrophoresis in $1.5 \%$ agarose gel stained with SYBR Green and photographed under ultraviolet light using the UV G:BOX (Syngene, USA). 
TABlE 1: Demographic and clinical characteristics of PFAPA patients.

\begin{tabular}{lc}
\hline Total number of patients & 81 \\
Male & $50(63 \%)$ \\
Female & $31(37 \%)$ \\
Age at disease onset (mean $\pm \mathrm{SD}$, & $2.1 \pm 1.5$ years $(0.1-6.5$ \\
range) & years) \\
Age at diagnosis (mean $\pm \mathrm{SD}$, range) & $3.9 \pm 1.7$ years $(1.2-7$ years) \\
$\begin{array}{l}\text { Interval between disease onset and } \\
\text { diagnosis (mean } \pm \mathrm{SD} \text {, range) }\end{array}$ & $1.9 \pm 1.5$ years $(0.4-6.5$ \\
$\begin{array}{l}\text { Duration of febrile episode (mean, } \\
\text { range) }\end{array}$ & years $)$ \\
$\begin{array}{l}\text { Interval between episodes (mean, } \\
\text { range) }\end{array}$ & 4.2 days (2-11 days) \\
\hline
\end{tabular}

2.6. Sanger Sequencing. PCR products $(2.5 \mu \mathrm{L})$ were purified using $1 \mu \mathrm{L}$ ExoSAP-IT enzyme mix (Affymetrix, USA), (incubation at $37^{\circ} \mathrm{C}$ for $15 \mathrm{~min}$; enzyme deactivation at $80^{\circ} \mathrm{C}$ for $15 \mathrm{~min}$ ) and subjected to direct nucleotide sequencing using the Big Dye Terminator cycle sequencing kit (Applied Biosystems, USA) following the manufacturer's instructions. The sequencing was performed on Applied Biosystems 3500 Genetic Analyser (Applied Biosystems, USA). Sequences were analyzed with the Sequencing Analysis Software v5.2.0 (Applied Biosystems, USA) and examined using the Nucleotide BLAST program.

All identified mutations/variants were validated in additional independent round of PCR and once again sequenced.

2.7. Genotyping PCR. The TaqMan (Applied Biosystems, USA) genotyping assays were used for two genetic variants that showed difference between frequency in PFAPA population and frequency in European or global population (MAF).

Genotyping experiments consisted of $10 \mathrm{ng}$ of double stranded DNA, 1x Master mix, and 0.5x of specific assay in a final reaction volume of $10 \mu \mathrm{L}$ and were run on the 7500 Fast RT-PCR system. The results were analyzed with the 7500 Software v2.0.1 (Applied Biosystems, USA).

2.8. Statistical Analysis. Descriptive statistics were reported as means $\pm \mathrm{SD}$ with ranges between extreme values added. Observed and expected frequencies of genetic variants were compared by using $\chi^{2}$ test.

\section{Results}

3.1. Clinical Characteristics. In the 7-year study period, 81 patients fulfilled criteria for the PFAPA syndrome. Table 1 describes the demographic and clinical characteristics of all PFAPA patients. The cohort consisted of 50 (63\%) boys and $31(37 \%)$ girls, with mean age at disease onset of $2.1 \pm 1.5$ years. Only 3 patients were older than 5 years at disease onset including twin sisters. Mean duration of febrile episode was 4.2 days with 4 -week interval between episodes. The longest afebrile period was 2 months in 2 patients.
TABLE 2: Signs and symptoms present in 81 PFAPA patients.

\begin{tabular}{lc}
\hline Symptom & $n(\%)$ \\
\hline Adenitis & $76(94 \%)$ \\
Pharyngitis & $79(98 \%)$ \\
Aphthous stomatitis & $45(56 \%)$ \\
\hline Abdominal pain & $41(51 \%)$ \\
Joint pain & $25(31 \%)$ \\
Vomiting & $33(41 \%)$ \\
Diarrhea & $18(22 \%)$ \\
Skin rash & $10(12 \%)$ \\
\hline
\end{tabular}

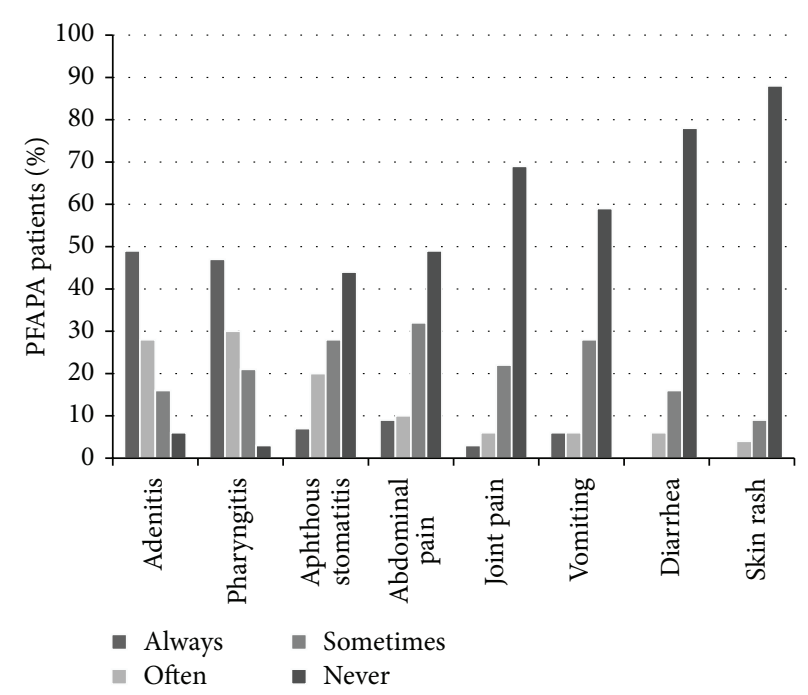

FIgURE 1: Frequencies of 8 clinical manifestations in 81 PFAPA patients.

All patients were asymptomatic during the afebrile period and showed normal growth and development.

3.2. Signs and Symptoms. Table 2 describes three main clinical manifestations (adenitis, pharyngitis, and aphthous stomatitis) and other signs accompanying fever in PFAPA patients. In three $(4 \%)$ children only one main symptom was present, 35 (43\%) patients had 2 symptoms, and 43 (53\%) had all 3 main symptoms present. Figure 1 shows frequencies (always, often, sometimes, and never) of 8 clinical manifestations in 81 PFAPA patients. In majority, patients parents reported onset of febrile episodes on a weekend (usually or often in 39/45 patients, never or sometimes in 6/45 patients).

3.3. Family History. In our PFAPA cohort 3 pairs of siblings were included: twin brothers, twin sisters, and two brothers. Family history of patients is presented in Table 3. Fifty out of $64(78 \%)$ patients had positive family history meaning that their first degree relatives had recurrent fevers in their childhood or tonsillectomy performed.

3.4. Laboratory Values. Inflammatory parameters were in all patients elevated during febrile episodes and normal during 
TABLE 3: Family history.

\begin{tabular}{lr}
\hline Recurrent fever or/and tonsillectomy in first degree relative & $50 / 64(78 \%)$ \\
\hline Recurrent fever or/and tonsillectomy in first degree relative, one family member affected only & $29 / 64(45 \%)$ \\
Recurrent fever or/and tonsillectomy in first degree relative, more than one family member affected & $21 / 64(38 \%)$ \\
\hline Tonsillectomy in first degree relative & $36 / 64(56 \%)$ \\
Recurrent fever without tonsillectomy in first degree relative & $14 / 64(22 \%)$ \\
\hline Tonsillectomy in second degree relative only* & $6 / 64(9 \%)$ \\
Negative & $8 / 64(13 \%)$ \\
Unknown & $17 / 81(21 \%)$ \\
\hline
\end{tabular}

${ }^{*}$ Excluding those with recurrent fever or tonsillectomy in first degree relative.

afebrile periods. Laboratory values of tested PFAPA patients are listed in Table 4.

3.5. Treatment. Of the 81 patients, 27 (33\%) used corticosteroids $(1-2 \mathrm{mg} / \mathrm{kg})$ at the onset of the febrile attack. They received two doses in 12-hour interval. Febrile attacks were successfully stopped in all patients. In 9/27 (33\%) patients the interval between the episodes was shortened after treatment with corticosteroids. Tonsillectomy was performed in $28(35 \%)$ patients and PFAPA resolved in 26/28 (93\%). Procedure was not successful in $2 / 28$ (7\%) patients, who subsequently continued to have episodes of PFAPA, although the episodes were shorter and less frequent.

3.6. Genetic Analyses. DNA of patients with PFAPA syndrome was analyzed for the 3 genes associated with monogenic periodic fever (NLRP3, MEFV, and $M V K$ ) and for the AIM2 gene.

3.7. AIM2 Gene. Whole coding region of AIM2 gene with promoter, $5^{\prime} \mathrm{UTR}$, and $3^{\prime} \mathrm{UTR}$ regions was analyzed in 62 patients. We identified 7 different variants: 1 novel and 6 already reported in dbSNP database (http://www.ncbi .nlm.nih.gov/SNP) with known allele frequency in general population. All variants found were in noncoding region of the AIM2 gene. Variants are listed in Table 5.

Genotyping assay for c.-208A $\geq \mathrm{C}$ variant, located in promoter region, was analyzed in 100 apparently healthy subjects. Variant was present in heterozygous state in 9 subjects, meaning that statistical significance between healthy subjects and PFAPA patients has not been reached (MAF case $/$ control $=0.06 / 0.045, P>0.05)$.

3.8. NLRP3 Gene. All variants listed here have codon number according to the number of the second methionine (Met) residue. We analyzed all coding regions of NLRP3 gene in 62 PFAPA patients and identified 4 different variants of possible clinical significance in $13(21 \%)$ patients. Variant Q703K in exon 3 was found in 9 patients; S726G in exon 4 in 1 patient; $\mathrm{P} 340 \mathrm{P}$ in exon 3 in 1 patient; and a novel variant $\mathrm{P} 200 \mathrm{~T}$ in exon 3 in 2 patients. Q703K variant (rs35829419) has frequency 0.058 in European population (MAF). We analyzed 100 apparently healthy subjects with genotyping assay for Q703K variant. Variant was present in heterozygous state in $12(12 \%)$ subjects meaning that statistical significance
TABLE 4: Laboratory parameters of PFAPA patients.

\begin{tabular}{lcc}
\hline Laboratory parameter & Mean \pm SD (range) \\
\hline IgA & $1.20 \pm 0.60 \mathrm{~g} / \mathrm{L}(0.29-3.03 \mathrm{~g} / \mathrm{L})$ \\
IgG & $10.18 \pm 2.41 \mathrm{~g} / \mathrm{L}(2.4-17.7 \mathrm{~g} / \mathrm{L})$ \\
IgM & $0.97 \pm 0.35 \mathrm{~g} / \mathrm{L}(0.35-1.76 \mathrm{~g} / \mathrm{L})$ \\
\hline Laboratory parameter & $<100 \mathrm{IE} / \mathrm{mL}$ & $n(\%)$ \\
\hline IgD & $>100 \mathrm{IE} / \mathrm{mL}$ & $9 / 48(81 \%)$ \\
Antinuclear antigen & Negative & $41 / 41(100 \%)$ \\
antibodies & Normal & $28 / 40(70 \%)$ \\
& Elevated & $10 / 40(25 \%)$ \\
Lymphocyte subsets & Low & $2 / 40(5 \%)$ \\
& ASO $<52 \mathrm{units} / \mathrm{mL}$ & $44 / 64(69 \%)$ \\
& $52<$ ASO $<500$ & $12 / 64(19 \%)$ \\
Antistreptolysin O & units/mL & $6 / 64(9 \%)$ \\
titer & $500<$ ASO $<1000$ & $2 / 64(3 \%)$ \\
& units/mL \\
& ASO $>1000 \mathrm{units} / \mathrm{mL}$ &
\end{tabular}

has not been reached (MAF case/control $=0.072 / 0.06, P>$ 0.05).

Variant S726G is a rare polymorphism (rs147946775) with MAF 0.002 in European population. Exon 4 of NLRP3 gene was analyzed also in this patient's parents and brother who are also carriers of this variant. Variant P200T, found in two unrelated patients, is a novel variant, but it is considered as a polymorphism according to Mutation Taster prediction program (http://www.mutationtaster.org/).

3.9. $M E F V$ Gene. All coding regions of the $M E F V$ gene were determined in 62 PFAPA patients. Six different variants were found in 5 (8\%) patients, namely, E148Q and A289V in exon 2, P369S and R408Q in exon 3, I591T in exon 9, and K695R in exon 10. All variants found were in a heterozygous state. One girl carried two variants, namely, P369S and R408Q in exon 3, and one boy was found to have I591T variant in $M E F V$ gene and Q703K in NLRP3 gene. Variant with unknown significance R202Q in exon 2 was found in 24 (39\%) patients in heterozygous state and in $6(10 \%)$ patients in homozygous state. 
TABLE 5: The list of all genetic variants of the PFAPA patients detected in AIM2 gene.

\begin{tabular}{lccc}
\hline Variant & rs number & Number of patients (variant in heterozygous state) & MAF global/MAF patients \\
\hline c. $-536 \mathrm{C}>\mathrm{T}$ & rs116289675 & 1 & $0.002 / 0.008$ \\
c. $-605 \mathrm{C}>\mathrm{A}$ & $\mathrm{New}$ & 1 & $\mathrm{NA} / 0.008$ \\
c. $-607 \mathrm{delA}$ & $\mathrm{rs} 3834102$ & 5 & $0.1152 / 0.04$ \\
c. $-208 \mathrm{~A}>\mathrm{C}$ & $\mathrm{rs} 41264459$ & $10+1$ homozygous & $0.028 / 0.097$ \\
c. $-28 \mathrm{~A}>\mathrm{G}$ & $\mathrm{rs} 2298803$ & 5 & $0.156 / 0.04$ \\
c. $-21+45 \mathrm{G}>\mathrm{C}$ & $\mathrm{rs} 41264457$ & 5 & $0.156 / 0.04$ \\
c. $-20-43 \mathrm{C}>\mathrm{T}$ & $\mathrm{rs} 74555135$ & 3 & $0.139 / 0.024$ \\
\hline
\end{tabular}

Variant nomenclature based on NCBI reference sequence NM_004833.1; MAF: minor allele frequency; NA: not available.

3.10. MVK Gene. All coding regions of $M V K$ gene were determined in 29 PFAPA patients and no variants of clinical significance have been identified.

In total, $17 / 62$ patients (27\%) carried 19 variants in $M E F V$ and NLRP3 genes. Other variants found in NLRP3, MEFV, and $M V K$ genes were polymorphisms with presumably no clinical significance and were present in general population in high frequency.

3.11. Genetic Associations with Clinical Features of the Syndrome. Patients who carry one of the variants experienced disease onset at a younger age compared to those without a variant present (mean $2.1 \pm 1.5$ years versus $2.3 \pm 1.6$ years) but had been diagnosed with PFAPA after a longer period of time (mean $2.6 \pm 2.5$ years versus $1.8 \pm 1.5$ years). Duration of the febrile episode was slightly shorter in patients with a variant (mean $4.1 \pm 1.6$ days versus $4.5 \pm 1.6$ days) and the interval between the episodes was longer (mean $4.2 \pm 1.8$ weeks versus $3.8 \pm 0.9$ weeks).

In total, $63 \%$ of patients with a variant presented with all 3 main PFAPA symptoms compared to $49 \%$ of patients without a variant. No difference was found in comparing positive family history (78\% versus $80 \%)$.

\section{Discussion}

PFAPA syndrome is the most common PFS of childhood with still unclear genetic background. So far no specific diagnostic test or laboratory markers are available for PFAPA and the diagnosis is based merely on diagnostic criteria and exclusion of other PFSs [10]. Since PFAPA syndrome has many overlapping features with monogenic PFSs, it has been suggested that it might have also a genetic origin. In this study, we clinically evaluated 81 PFAPA patients; performed analysis of AIM2, MEFV, NLRP3, and MVK genes in 62 patients; and assessed possible genetic correlations with clinical features.

We report a large cohort of PFAPA patients longitudinally followed for almost 7 years in a single tertiary center. Males were the majority $(63 \%)$, which is similar to previous studies $[6,8,17]$. During the fever episode adenitis and pharyngitis were present in most of the patients (94\% and 98\%, resp.) and aphthous stomatitis was present in $56 \%$ of the patients, which is similar to other studies [5-8, 17-19]. All three major PFAPA symptoms were present in $53 \%$ of patients. This result is higher comparing to published data reporting the occurrence of all three symptoms in $28 \%$ of patients [17]. Seventy-nine percent of our patients were tested for ASO titer. Among them, $87 \%$ had negative ASO titers indicating that they did not encounter streptococcal infection in the past. To the best of our knowledge, there are no published data about ASO titers in PFAPA patients so far. According to our experience ASO titer could be a very helpful tool in differentiating PFAPA patients from patients suffering from recurrent streptococcal infections with similar clinical picture.

Optimal treatment for patients with PFAPA is uncertain and considering unknown etiology of the disease it is only symptomatic [20]. Given the fact that PFAPA is selflimiting disease, treatment of any kind is still a matter of debate. Corticosteroid therapy usually aborts an episode within hours, but it does not prevent future fever flares $[3,6]$. Thirty-three percent of patients in our cohort were receiving corticosteroids at the beginning of febrile attacks with successful abortion of the attack in all of them.

Tonsillectomy in PFAPA patients is still controversial considering that affected children usually recover spontaneously after few years and the procedure itself is invasive with unknown long term effect for the patients. However, the method is effective and usually completely stops the attacks [21]. In our cohort $37 \%$ of patients underwent tonsillectomy with complete remission in $93 \%$ of them. This finding is similar to those reported in other studies where complete remission was up to $100 \%[19,21]$.

PFAPA is classically considered as a sporadic and noninherited syndrome [8]; however, several studies reported PFAPA patients with family members having recurrent fever in their childhood $[11,12,22]$. In our cohort, we identified 3 pairs of siblings. Moreover, positive family history was found in 50/64 (78\%) patients, which is the highest rate ever reported. Additionally, 21/64 (37\%) of our patients had more than one first degree family member affected, clearly indicating possible familial association. In a recent study positive family history (recurrent fever) has been reported in 38/84 (45\%) affected patients; additionally, 10 of these patients had a family member with diagnosed PFAPA syndrome [10]. In another study authors compared PFAPA patients with complete remission before puberty (mean duration of symptoms: 6.3 years) and those with remission after 18 years. Family history in the first and the second groups was positive in $40 \%$ and $4 \%$, respectively [18]. Although no causative gene has been identified for the PFAPA syndrome, these findings 
clearly suggest genetic background rather than sporadic background. Alternatively, affected family members could be exposed to the same infection or any other environmental trigger which could ultimately lead to the development of clinical manifestations of PFAPA syndrome.

The cause and etiology of PFAPA is still unknown. Due to a sudden response to a single dose of corticosteroids PFAPA symptoms may be caused by dysregulated inflammatory cytokines production rather than by infection [23]. Studies on cytokines showed rapid rise and fall of proinflammatory cytokines IL- $1 \beta$, IL-18, TNF- $\alpha$, and IFN- $\gamma$ in the early hours of flare and rise of IL- 6 in the later state of the flare, with the normalization in the afebrile state of the disease [24], whereas T-cell associated IL-7, IL-17 [24], and anti-inflammatory cytokines IL-10 and IL-4 were decreased during flare [1].

IL-1 $\beta$ plays an important role in all autoinflammatory syndromes including PFAPA. Recent study strongly suggests that IL- $1 \beta$ monocyte production is dysregulated in patients with PFAPA syndrome as a consequence of alteration in inflammasome activation probably linked to genetic defect in inflammasome related genes [9]. Additionally, increased expression of IL-1 and inflammasome associated genes (AIM2, CASP1) was reported during PFAPA flare. Furthermore, therapeutic efficacy of IL- $1 \beta$ blockade on resolution of the disease flare was reported [5]. All these findings strongly indicate involvement of inflammasome related genes in PFAPA pathogenesis.

Therefore, we analyzed two inflammasome genes, whole coding region of AIM 2 gene with promoter, $5^{\prime} \mathrm{UTR}$, and $3^{\prime}$ UTR regions included, and NLRP3 gene in 62 patients. In the AIM2 gene 7 variants had been identified, 1 novel and 6 already reported, all located in noncoding region of the gene. Five of these variants had in our PFAPA cohort similar or smaller allele frequency compared to general population and it is less likely to have a significant role in the PFAPA pathogenesis according to in silico analyses we performed. Variant c. $-208 \mathrm{~A} \geq \mathrm{C}$ (rs41264459) is located in the promoter region of the gene; therefore, it could have impact on gene level expression. MAF of this variant in the general population is 0.03 . In our study, we obtained MAF 0.06 in the patient cohort and MAF 0.045 in the healthy control group, which were not statistically significantly different. Although no connection was identified between the AIM2 gene variants and PFAPA syndrome, it is still possible that epigenetic or somatic alterations of the gene could play a role in the disease onset.

Of the 62 PFAPA patients, 13 (21\%) carried one of the four different NLRP3 variants identified in this cohort. Mutations in NLRP3 gene are known to cause cryopyrin-associated periodic fever syndromes (CAPS). NLRP3 gene encodes cryopyrin, a component of the NLRP3 inflammasome that activates caspase- 1 leading to inflammation by excessive production of IL- $1 \beta[15,25]$. The most common variant in our cohort was Q703K, which was found in 9 patients (MAF 0.072) and in 12 apparently healthy individuals (MAF 0.06). Similar rates were recently reported in a study of 57 PFAPA patients in which the Q703K variant was found in 9 patients [9]. Role of this variant in CAPS syndrome is still unclear; it is considered as either a low-penetrance mutation or a polymorphism without functional effect [26]. Although statistical significance has not been reached, this does not mean that Q703K variant does not have an effect on inflammasome activation [9]. A recent study showed that the Q703K variant is a gain-of-function alteration leading to an overactive NLRP3 inflammasome [27]. Variant S726G which is found in one of our PFAPA patients is a rare polymorphism that was previously described in two Turkish patients with typical CAPS phenotype [28]. This variant is listed in the Infevers database (http://fmf.igh.cnrs.fr/ISSAID/infevers/) as a symptomatic. Mother and a brother of our patient are both carriers of this variant but did not exhibit clinical manifestations of PFAPA, so we suspect that it is not disease causing. P340P which is found in one patient is a synonymous variant, listed in the Infevers database and in HGMD database (http://www.hgmd.org/) as a disease causing mutation, but with unknown functional effect.

Variant c.598C $>$ A which changes aminoacid proline at position 200 into threonine (P200T) was found in two unrelated patients. It is a novel variant and is considered as a polymorphism according to Mutation Taster prediction program (http://www.mutationtaster.org/). The analysis with the Variant Effect Predictor (http://www.ensembl.org/ Homo_sapiens/Tools/VEP) did not provide any additional information on how this variant could influence the function of the NLRP3 gene. Unfortunately, DNA from the parents of either patient was not available for further genetic analysis to see whether this variant was inherited or occurred de novo. Nevertheless, the presence of this variant in 2 patients indicates its importance in PFAPA phenotype.

Total of $5(8 \%)$ patients carried 6 different variants in MEFV gene, namely, E148Q, A289V, P369S, R408Q, I591T, and K695R. All variants found are listed in Infevers database as variants found in patients with familial Mediterranean fever (FMF). However, due to the absence of functional tests, the consequences of these variants on $M E F V$ gene function and structure remain unknown. The role of E148Q variant is uncertain, whether it is mildly pathogenic with reduced penetrance or a variant without the causative role $[9,29]$. K695R and P369S variants have also been reported as having reduced penetrance [30].

Recent study on predominant mutations in $M E F V$, CARD15/NOD2, TNFr1A, and NLRP3 genes in patients diagnosed with PFAPA syndrome showed that 19 of 57 patients carried one or more of the variants tested, with $M E F V$ gene variants being found in 16 patients [13]. In another study authors evaluated variants in 4 autoinflammatory genes (NLRP3, MEFV, TNFRSF1A, and MVK) in total of 57 patients, where they identified variants in 15 patients, with NLRP3 variants found in 12 patients [9]. In spite of the high frequency of these variants, authors could not conclude whether these variants are associated with PFAPA or not.

\section{Conclusions}

PFAPA is classically considered as a sporadic and noninherited syndrome, but extremely high rate of positive family history in our cohort clearly indicates possible genetic 
background of the disease. Total of 17 patients (27\%) carried variants in $M E F V$ and NLRP3 genes. These are all probably low-penetrant variants which are usually not confirmative for FMF or CAPS but could play a role in susceptibility to autoinflammation and as such they might also play a role in the pathogenesis of PFAPA. We hypothesize that PFAPA could be the result of multiple low-penetrant gene variants in different genes in combination with epigenetic and environmental factors leading to one uniform clinical picture.

\section{Conflict of Interests}

The authors declare no conflict of interests.

\section{Acknowledgments}

This work was partially supported by the Slovenian Research Agency Grants L3-4150 and P3-0343 and by the University Medical Center, Ljubljana, Research Grant 20120208.

\section{References}

[1] S. Stojanov, F. Hoffmann, A. Kéry et al., "Cytokine profile in PFAPA syndrome suggests continuous inflammation and reduced anti-inflammatory response," European Cytokine Network, vol. 17, no. 2, pp. 90-97, 2006.

[2] A. Marcuzzi, E. Piscianz, G. Kleiner et al., "Clinical genetic testing of periodic fever syndromes," BioMed Research International, vol. 2013, Article ID 501305, 8 pages, 2013.

[3] S. Padeh, "Periodic fever syndromes," Pediatric Clinics of North America, vol. 52, no. 2, pp. 577-609, 2005.

[4] S. L. Masters, A. Simon, I. Aksentijevich, and D. L. Kastner, "Horror autoinflammaticus: the molecular pathophysiology of autoinflammatory disease," Annual Review of Immunology, vol. 27, pp. 621-668, 2009.

[5] S. Stojanov, S. Lapidus, P. Chitkara et al., "Periodic fever, aphthous stomatitis, pharyngitis, and adenitis (PFAPA) is a disorder of innate immunity and Th1 activation responsive to IL-1 blockade," Proceedings of the National Academy of Sciences of the United States of America, vol. 108, no. 17, pp. 7148-7153, 2011.

[6] S. Padeh, N. Brezniak, D. Zemer et al., "Periodic fever, aphthous stomatitis, pharyngitis, and adenopathy syndrome: clinical characteristics and outcome," The Journal of Pediatrics, vol. 135, no. 1, pp. 98-101, 1999.

[7] G. S. Marshall, K. M. Edwards, J. Butler, and A. R. Lawton, "Syndrome of periodic fever, pharyngitis, and aphthous stomatitis," The Journal of Pediatrics, vol. 110, no. 1, pp. 43-46, 1987.

[8] K. T. Thomas, H. M. Feder Jr., A. R. Lawton, and K. M. Edwards, "Periodic fever syndrome in children," The Journal of Pediatrics, vol. 135, no. 1, pp. 15-21, 1999.

[9] L. Kolly, N. Busso, A. von Scheven-Gete et al., "Periodic fever, aphthous stomatitis, pharyngitis, cervical adenitis syndrome is linked to dysregulated monocyte IL- $1 \beta$ production," The Journal of Allergy and Clinical Immunology, vol. 131, no. 6, pp. 1635-1643, 2013.

[10] M. Cochard, J. Clet, L. Le et al., "PFAPA syndrome is not a sporadic disease," Rheumatology, vol. 49, no. 10, pp. 1984-1987, 2010.
[11] A. Z. Akelma, M. N. Cizmeci, M. K. Kanburoglu et al., "Is PFAPA syndrome really a sporadic disorder or is it genetic?" Medical Hypotheses, vol. 81, no. 2, pp. 279-281, 2013.

[12] P. Antón-Martín, R. O. Movilla, S. G. Martín et al., "PFAPA syndrome in siblings. Is there a genetic background?" European Journal of Pediatrics, vol. 170, no. 12, pp. 1563-1568, 2011.

[13] E. Dagan, R. Gershoni-Baruch, I. Khatib, A. Mori, and R. Brik, "MEFV, TNF1rA, CARD15 and NLRP3 mutation analysis in PFAPA," Rheumatology International, vol. 30, no. 5, pp. 633-636, 2010.

[14] V. Hornung, A. Ablasser, M. Charrel-Dennis et al., "AIM2 recognizes cytosolic dsDNA and forms a caspase-1-activating inflammasome with ASC," Nature, vol. 458, no. 7237, pp. 514518, 2009.

[15] K. Schroder and J. Tschopp, “The Inflammasomes," Cell, vol. 140, no. 6, pp. 821-832, 2010.

[16] T. Fernandes-Alnemri, J.-W. Yu, P. Datta, J. Wu, and E. S. Alnemri, "AIM2 activates the inflammasome and cell death in response to cytoplasmic DNA," Nature, vol. 458, no. 7237, pp. 509-513, 2009.

[17] D. Tasher, E. Somekh, and I. Dalal, "PFAPA syndrome: new clinical aspects disclosed," Archives of Disease in Childhood, vol. 91, no. 12, pp. 981-984, 2006.

[18] V. M. Wurster, J. G. Carlucci, H. M. Feder, and K. M. Edwards, "Long-term follow-up of children with periodic fever, aphthous stomatitis, pharyngitis, and cervical adenitis syndrome," The Journal of Pediatrics, vol. 159, no. 6, pp. 958-964, 2011.

[19] H. M. Feder and J. C. Salazar, "A clinical review of 105 patients with PFAPA (a periodic fever syndrome)," Acta Paediatrica, vol. 99, no. 2, pp. 178-184, 2010.

[20] G. Vigo and F. Zulian, "Periodic fevers with aphthous stomatitis, pharyngitis, and adenitis (PFAPA)," Autoimmunity Reviews, vol. 12, no. 1, pp. 52-55, 2012.

[21] E. Galanakis, C. E. Papadakis, E. Giannoussi, A. D. Karatzanis, M. Bitsori, and E. S. Helidonis, "PFAPA syndrome in children evaluated for tonsillectomy," Archives of Disease in Childhood, vol. 86, no. 6, pp. 434-435, 2002.

[22] M. Adachi, A. Watanabe, A. Nishiyama et al., "Familial cases of periodic fever with aphthous stomatitis, pharyngitis, and cervical adenitis syndrome," The Journal of Pediatrics, vol. 158, no. 1, pp. 155-159, 2011.

[23] S. Padeh, N. Stoffman, and Y. Berkun, "Periodic fever accompanied by aphthous stomatitis phayngitis and cervical adenitis syndrome (PFAPA syndrome) in adults," The Israel Medical Association Journal, vol. 10, no. 5, pp. 358-360, 2008.

[24] K. L. Brown, P. Wekell, V. Osla et al., "Profile of blood cells and inflammatory mediators in periodic fever, aphthous stomatitis, pharyngitis and adenitis (PFAPA) syndrome," BMC Pediatrics, vol. 10, article 65, 2010.

[25] A. Insalaco, G. Prencipe, P. S. Buonuomo et al., "Case report: a novel mutation in the associated with an unexpected phenotype of CAPS," Clinical and Experimental Rheumatology, vol. 32, no. 1, pp. 123-125, 2013.

[26] A. Vitale, O. M. Lucherini, M. Galeazzi, B. Frediani, and L. Cantarini, "Long-term clinical course of patients carrying the Q703K mutation in the NLRP3 gene: a case series," Clinical \& Experimental Rheumatology, vol. 30, no. 6, pp. 943-946, 2012.

[27] D. Verma, E. Särndahl, H. Andersson et al., “The Q705K polymorphism in NLRP3 is a gain-of-function alteration leading to excessive interleukin-1 $\beta$ and IL-18 production," PLOS ONE, vol. 7, no. 4, Article ID e34977, 2012. 
[28] A. Berdeli, S. Nalbantoglu, D. Tigli, I. Demirel, M. Atan, and B. Sozeri, "PW02-028-association of novel NLRP3 mutations with CAPS phenotype in Turkish patients," Pediatric Rheumatology, vol. 11, supplement 1, article A169, 2013.

[29] R. Topaloglu, C. Yildiz, E. Taskiran et al., "PW01-004-the sequence analysis in E148Q homozygous patients," Pediatric Rheumatology Online Journal, vol. 11, supplement 1, article A57, 2013.

[30] I. Aksentijevich, Y. Torosyan, J. Samuels et al., "Mutation and haplotype studies of familial Mediterranean fever reveal new ancestral relationships and evidence for a high carrier frequency with reduced penetrance in the Ashkenazi Jewish population," The American Journal of Human Genetics, vol. 64, no. 4, pp. 949$962,1999$. 


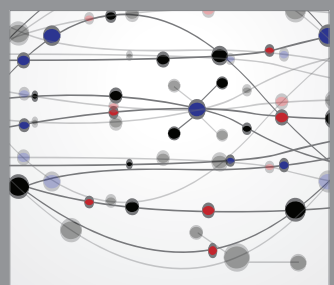

The Scientific World Journal
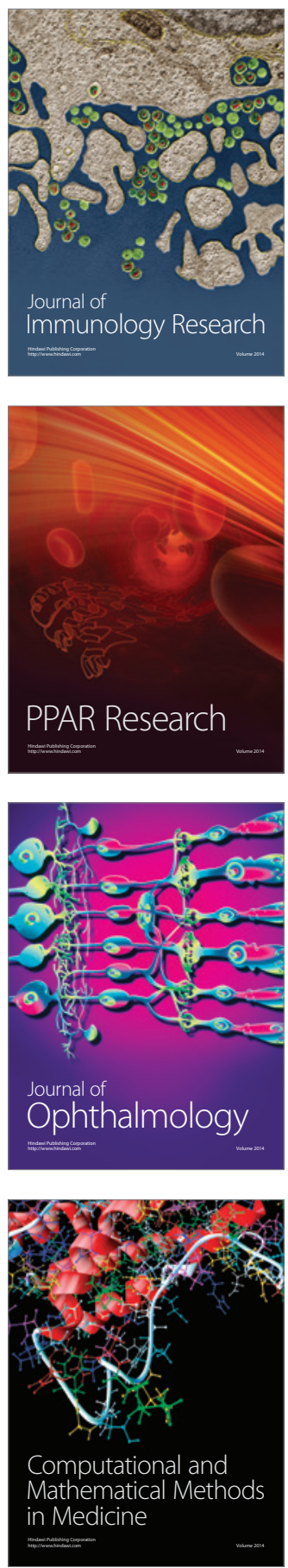

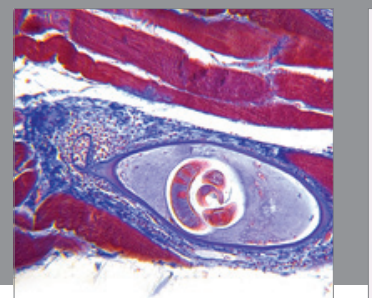

Gastroenterology

Research and Practice
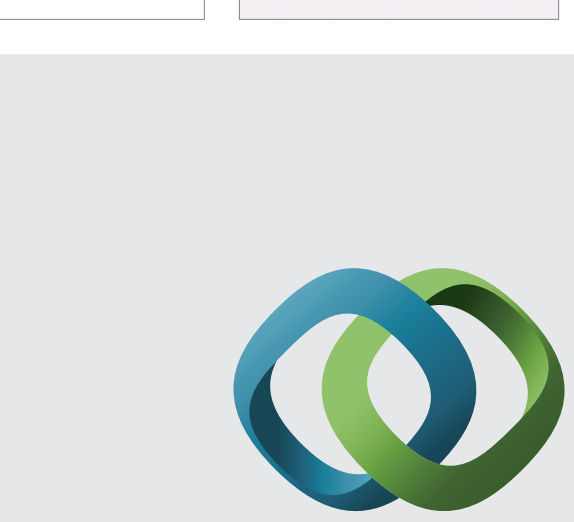

\section{Hindawi}

Submit your manuscripts at

http://www.hindawi.com
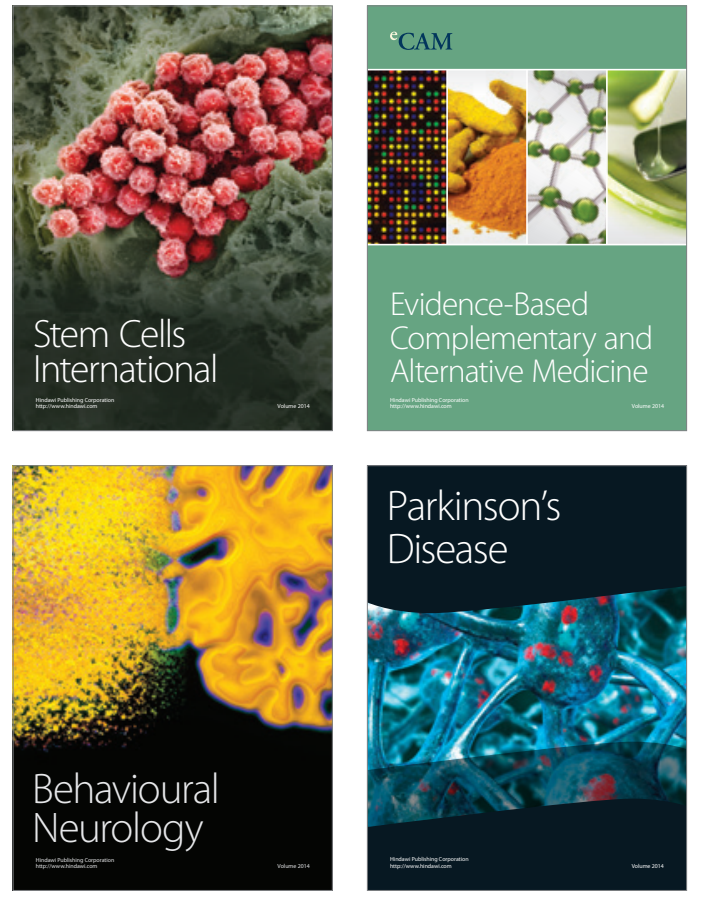
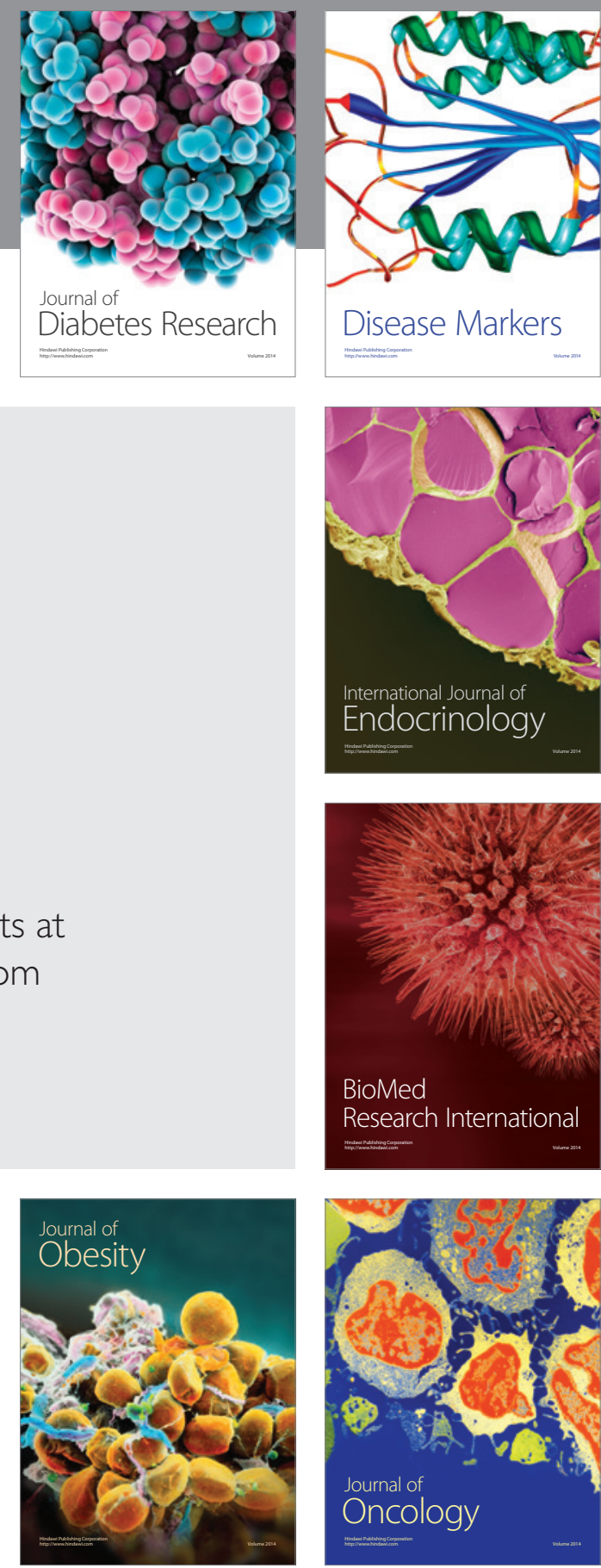

Disease Markers
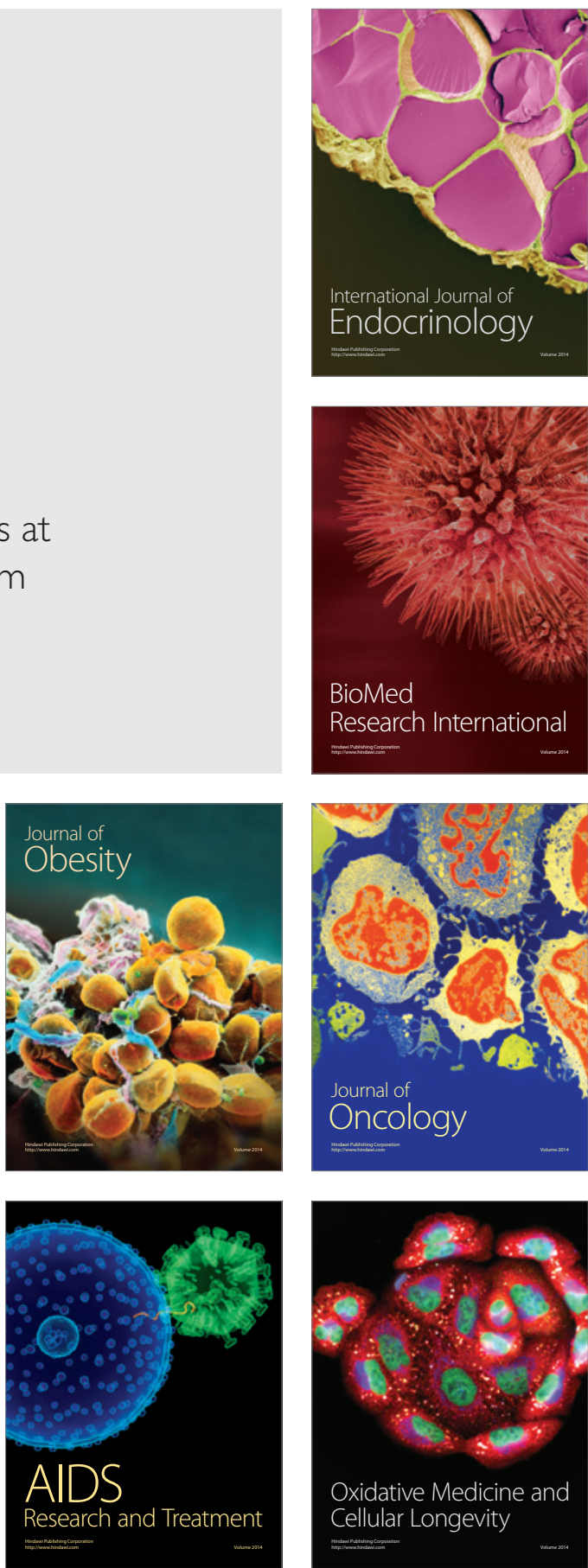